To our knowledge this is the first report of recurrent anaemia without frank gastrointestinal haemorrhage as a presentation of cholesterol embolism. This elderly male patient, athough not hypertensive, had many risk factors for this disease: he was a diabetic, an ex-smoker and had evidence of generalized atherosclerosis. We cannot be certain in retrospect for how long cholesterol embolism in the superior mesenteric axis was the source of blood loss, but right hemicolectomy appeared to be curative in this case and no other cause for bleeding was found on close examination of the specimen. The interval of one month between

\section{References}

1. Fine, M.J., Kapoor, W. \& Falanga, V. Cholesterol crystal embolization: a review of 221 cases in the English literature. Angiology 1987, 38: 769-784.

2. Socinski, M.A., Frankel, J., Morrow, P.L. \& Krawitt, L. Painless diarrhoea secondary to intestinal ischaemia. Diagnosis of atheromatous emboli by jejunal biopsy. Dig Dis $\mathrm{Sci}$ 1984, 29: 674-677.

3. Mulliken, J.B. \& Bartlett, M.K. Small bowel obstruction secondary to atheromatous embolism: a case report and review of the literature. Ann Surg 1971, 174: 145-150.

4. Blundell, J.W. Small bowel stricture secondary to multiple cholesterol emboli. Histopathology 1988, 13: 459-462.

5. Francis, J. \& Kapoor, W.N. Intestinal pseudopolyps and gastrointestinal haemorrhage due to cholesterol crystal embolization. Am J Med 1988, 85: 269-271.

6. Cross, S.S. How common is cholesterol embolism? J Clin Pathol 1991, 44: 859-861.

7. Dahlberg, P.J., Frecentese, D.F. \& Cogbill, T.H. Cholesterol embolism: experience with 22 histologically proven cases. Surgery 1989, 105: 737-746. angiography and surgery makes it unlikely that the ischaemic changes observed were due to cholesterol embolism at aortic instrumentation. It is possible that the episode of small bowel obstruction was also a sequel of cholesterol embolism, since the healing process following an episode of extensive mucosal ischaemia can result in concentric fibrosis and narrowing of the bowel lumen. ${ }^{3}$

This case illustrates that cholesterol embolism should be considered as a possible cause of unexplained gastrointestinal blood loss in an elderly patient with atherosclerosis.

8. Queen, M., Biem, H.J., Moe, G.W. \& Sugar, L. Development of cholesterol embolization after intravenous streptokinase for acute myocardial infarction. Am J Cardiol 1990, 65: 1042-1043.

9. Farouhar, F.A., Mohit, M., Gardner, P. \& Smith, N. Cholesterol embolism causing bleeding gastric ulcers. Ann Clin Lab Sci 1988, 18: 260-265.

10. Moolenaar, W., Kreuning, J., Eulderink, F. \& Lamers, C. Ischaemic colitis and acalculous necrotising cholecystitis as rare manifestations of cholesterol emboli in the same patient. Am J Gastroenterol 1989, 84: 1421-1422.

11. O'Briain, D.S., Jeffers, M., Kay, E.W. \& Hourihane, D. O'B. Bleeding due to colorectal atheroembolism: diagnosis by biopsy of adenomatous polyps or of ischaemic ulcer. $A m J$ Surg Pathol 1991, 15: 1078-1082.

12. Chan, T., Levine, M.S. \& Park, Y. Cholesterol embolization as a cause of caecal infarct mimicking carcinoma. Am $J$ Radiol 1988, 150: 1315-1316.

\title{
Toxic megacolon with late perforation in Campylobacter colitis - a cautionary tale
}

\author{
Samir K. Vyas, Nicholas N. Law ${ }^{1}$, Simon Hill ${ }^{2}$ and Christian A. Loehry ${ }^{3}$ \\ Department of Clinical Medicine, Royal Bournemouth General Hospital, East Dorset, ${ }^{1}$ Department of \\ Microbiology, Poole General Hospital, West Dorset, and ${ }^{2}$ Department of General Surgery, Royal \\ Bournemouth General Hospital, East Dorset, UK
}

Summary: We report a case of Campylobacter-induced colitis complicated by toxic megacolon which led to colonic perforation requiring a total colectomy and ileostomy. 


\section{Introduction}

Campylobacter enteritis is now recognized as the commonest infectious cause of diarrhoea in the UK. The illness is usually self-limiting and lifethreatening complications are rare. We describe a case of severe Campylobacter colitis presenting with toxic megacolon in a previously healthy young woman. This was complicated by late colonic perforation requiring a subtotal colectomy despite early treatment with antibiotics and intravenous steroids while the aetiology was unclear.

\section{Case history}

A previously fit 38 year old woman presented with a 4 day history of vague abdominal discomfort, fever, rigors and loose, watery diarrhoea. Her bowels opened up to 12 times per day with blood and pus in the stool. There had been no previous similar bowel disturbance nor had she received antibiotics just prior to this illness. On examination she was febrile $\left(38^{\circ} \mathrm{C}\right)$, tachycardic $(120$ beats/ minute, regular) and severely dehydrated $(25 \mathrm{mmHg}$ postural drop in systolic blood pressure). The abdomen was generally distended and tender, but there was no guarding.

Sigmoidoscopy showed an inflamed, friable rectal mucosa with overlying pus and contact bleeding. The abdominal radiograph showed a markedly dilated, empty colon with a maximum diameter of $6.7 \mathrm{~cm}$ in the transverse segment. There was widespread thickening of the large bowel wall and loss of the normal haustral pattern (Figure 1).

A provisional diagnosis of toxic megacolon secondary to infection or inflammatory bowel disease was made. Intravenous rehydration, hydrocortisone and oral ciprofloxacin were commenced. After 4 days, a rectal biopsy was reported as showing an abundance of acute inflammatory cells in the lamina propria, but no cryptitis, crypt abscesses, goblet cell depletion or granulomata were seen. Campylobacter jejuni was cultured from three stool samples but Clostridium difficile or its toxin were not present. Serial blood cultures remained sterile. The hydrocortisone was therefore discontinued.

Her general condition improved over 10 days with resolution of her hypotension, tachycardia, abdominal distension and tenderness. Bowel frequency decreased to three stools per day with occasional blood and pus. However, the haemoglobin concentration dropped from $13.2 \mathrm{~g} / 1$ to $10.5 \mathrm{~g} / \mathrm{l}$. The serum albumin concentration fell from $36 \mathrm{~g} / 1$ to $24 \mathrm{~g} / \mathrm{l}$, despite parenteral nutrition for the last 5 days.

On the eleventh day, she developed severe abdominal pain, tachycardia and increased epigas- tric tenderness with guarding. Subhepatic free gas was present on the abdominal radiograph with a toxic megacolon (Figure 2).

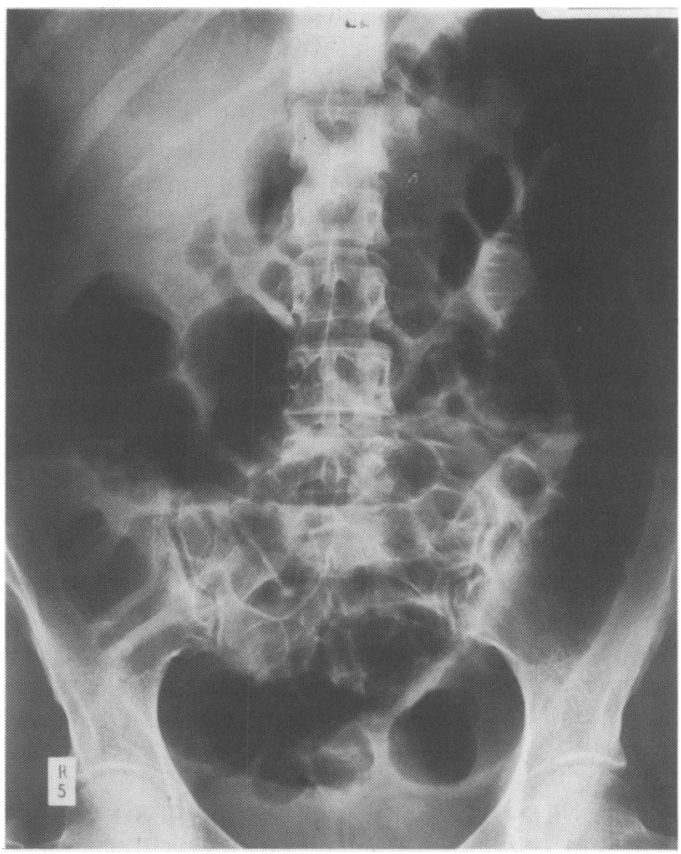

Figure 1 Abdominal radiograph at presentation.

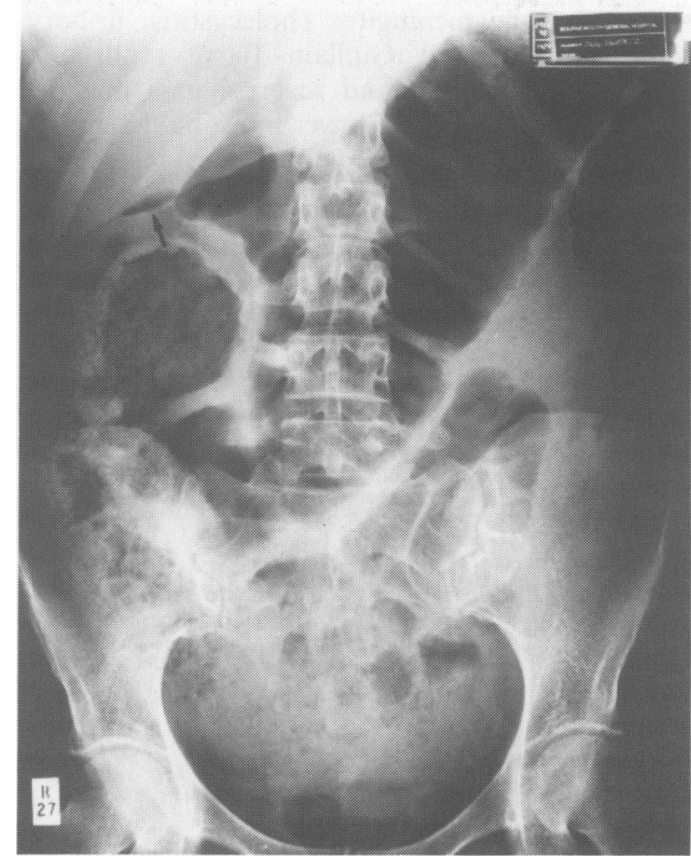

Figure 2 Abdominal radiograph the eleventh day showing gross colonic dilatation on subhepatic free air (arrow). 
Emergency laparotomy confirmed a very dilated colon which was friable and oedematous, particularly in the transverse segment. The caecum and sigmoid colon were perforated with a fibrinous exudate over the transverse colon. A total colectomy was performed leaving an ileostomy and mucous fistula.

Histological examination of the excised colon, together with $4.5 \mathrm{~cm}$ of terminal ileum showed extensive mucosal ulceration and inflammation, but where the mucosa was preserved, there was minimal cryptitis or goblet cell depletion. Lymph nodes showed severe reactive hyperplasia. These findings were consistent with severe acute Campylobacter colitis.

Postoperatively, she was treated with intravenous cefuroxime, erythromycin and metronidazole and she made an unremarkable recovery. Her liver function tests returned to normal after 14 days. She remains well 8 months later and a reversal of ileostomy with ileo-rectal anastomosis is planned in the near future.

\section{Discussion}

Campylobacter enteritis is increasingly common with over 32,000 cases reported in England and Wales in 1989, a total which has quadrupled since 1979. ${ }^{1}$ In the vast majority a relatively mild, self-limiting gastroenteritis is observed, hardly requiring any specific treatment. Atypical manifestations include meningitis, cholecystitis, urinary tract infection and Guillain-Barré syndrome. Rarely, a protracted and severe illness may be complicated by massive lower intestinal haemorrhage $^{2}$ or acute colitis mimicking a relapse of inflammatory bowel disease with toxic megacolon. ${ }^{3}$

Toxic megacolon due to Campylobacter colitis usually responds to appropriate antibiotics and supportive treatment, ${ }^{3}$ although it is possible that cases diagnosed after colonic resection may be

\section{References}

1. Skirrow, M.B. Foodborne illness, Campylobacter. Lancet 1990, 336: 921-923.

2. Michalk, D.M., Perault, J., Gilchrist, M.J., Dozois, R.R. Carney, J.J. \& Sheedy, P.F. Campylobacter fetus ss. jejuni; a cause of massive lower gastrointestinal haemorrhage. Gastroenterology 1980, 79: 742-745.

3. Anderson, J.B., Tanner, A.H. \& Brobribb, A.J.M. Toxic megacolon due to Campylobacter colitis. Int J Color Dis 1986, under-reported. We could find only one previous case of perforation following Campylobacterinduced toxic megacolon. ${ }^{3}$ In this, as in our case, intravenous corticosteroids and broad spectrum antibiotics were administered.

Parenteral hydrocortisone and oral ciprofloxacin, a 5-amino fluoroquinolone antibacterial agent, were employed empirically in our case until stool cultures and rectal histology became available. Whilst acknowledging the risk of exacerbating or masking an infectious diarrhoea by treatment with intravenous corticosteroids, we believe their use was justified in the initial management of toxic megacolon.

There is general acceptance to treat with antibiotics those patients with high fever, bloody diarrhoea of more than eight stools per day or persistent symptoms for more than one week. Most strains of Campylobacter jejuni are sensitive to ciprofloxacin or erythromycin.

Fluoroquinolone resistance has been reported in isolates from human stools in one series from the Netherlands in which 16 of 145 isolates $(11 \%)$ were resistant (MIC $4 \mathrm{mg} / \mathrm{l}$ ). ${ }^{4}$ The presence of erythromycin-resistant strains has varied up to $8 \%$ in Sweden ${ }^{5}$ and have been described in the UK. ${ }^{4}$ The isolates from our patient's stools, however, were sensitive to ciprofloxacin and erythromycin? The fact that this illness was accompanied b. anaemia, hypoproteinaemia and a transient rise i⿺辶̄ liver enzymes suggested that the infection was severe and that complications might arise.

We conclude on a cautionary note in the management of an increasingly common condition which is usually self-limiting but which can produce life-threatening complications.

\section{Acknowledgements}

We are grateful to Mr John Trapnell for his permission to report this case and to Mrs Barbara Thomas for typing the manuscript.

4. Burridge, R.D. \& Phillips, I. Erythromycin resistant Campylobacter jejuni. J Antimicrob Chemother 1984, 14: 307.

5. Walder, M. \& Forsgren, A. Erythromycin resistant Cam- O pylobacters. Lancet 1978, ii: 1201. 